ISSN 0103-5150

Fisioter. Mov., Curitiba, v. 25, n. 2, p. 445-451, abr./jun. 2012 Licenciado sob uma Licença Creative Commons

\title{
A importância da estabilização central no método Pilates: uma revisão sistemática
}

\author{
The importance of central stabilization in \\ Pilates method: a systematic review
}

\section{Gisele Marés ${ }^{[\mathrm{a}]}$, Keti Batista de Oliveira ${ }^{[\mathrm{b}]}$, Marcia Carla Piazza ${ }^{[\mathrm{cl}}$, Cássio Preis ${ }^{[\mathrm{d}]}$, Luiz Bertassoni Neto ${ }^{[\mathrm{e}]}$}

[a] Fisioterapeuta, Especialista em Ortopedia, Traumatologia e Desportiva pela Pontifícia Universidade Católica do Paraná (PUCPR), São Francisco do Sul, SC - Brasil, e-mail: gisele_mares@hotmail.com

[b] Fisioterapeuta, Especialista em Ortopedia, Traumatologia e Desportiva pela Pontifícia Universidade Católica do Paraná (PUCPR), São Francisco do Sul, SC - Brasil, e-mail: keti.fisio@yahoo.com.br

[c] Fisioterapeuta, Especialista em Ortopedia, Traumatologia e Desportiva pela Pontifícia Universidade Católica do Paraná (PUCPR), Timbó, SC - Brasil, e-mail: marciapiazza@yahoo.com.br

[d] Orientador, coordenador da Especialização em Fisioterapia Ortopédica, Traumatológica e Desportiva, e responsável pelo Centro de Dinamometria Isocinética e Baropodometria, Clínica de Fisioterapia, Pontifícia Universidade Católica do Paraná (PUCPR), Curitiba, PR - Brasil, e-mail: cassiopreis@hotmail.com

[e] Orientador, docente da Pontifícia Universidade Católica do Paraná (PUCPR), Curitiba, PR - Brasil, e-mail: luizbertassoni@yahoo.com.br

\section{Resumo}

Introdução: Joseph Humbertus Pilates criou uma série de exercícios baseados nos movimentos progressivos que o corpo é capaz de executar, os quais envolvem contrações isotônicas e, principalmente, isométricas, no que denominou power house (ou centro de força). Objetivos: Este estudo tem como objetivo geral analisar os aspectos relacionados à importância da estabilização central no método Pilates. Materiais e métodos: Foi realizada uma revisão sistemática sobre o assunto na biblioteca central e virtual da Pontifícia Universidade Católica do Paraná, e também por meio do site de busca BIREME acessando as bases de dados LILACS, PUB MED, CAPES e MEDLINE. A pesquisa abrangeu livros, revistas, periódicos publicados entre 2000 e 2010, e os descritores utilizados para a busca foram pilates e core. Resultados: Concluiu-se que a estabilização central ajuda o indivíduo a obter ganhos de força, controle neuromuscular, potência e resistência muscular, facilitando um equilibrado funcionamento muscular. Conclusão: Embora seja necessária 
uma bibliografia mais extensa, pode-se verificar que a estabilização central no Pilates é importante para o equilíbrio de carga apropriado dentro da coluna vertebral, pélvis.

Palavras-chave: Fisioterapia. Estabilização central. Core. Pilates.

\section{Abstract}

Introduction: Humbertus Joseph Pilates created a series of exercises based on the progressive movements that the body is able to perform, which involve isotonic contractions and mainly isometric, in what he called power house (or center of force). Objectives: This study aims at analyzing the aspects related to the central importance of stabilizing in the Pilates method. Materials and methods: We performed a systematic review in the central and virtual library of Pontifical Catholic University of Parana, and also through the research website BIREME, by accessing the following databases: LILACS, PUB MED, CAPES, MEDLINE and LILACS. The research covered books, magazines and journals published between 2000 and 2010, and the descriptors used for the search were Pilates and core. Results: The central stabilization helps the individual to gain strength, neuromuscular control, power and muscular endurance, which facilitates a balanced muscle function. Conclusion: Although we need a greater number of references, one can verify that the Pilates core stabilization is important for the proper load balance within the spine, pelvis.

Keywords: Physiotherapy. Central stabilization. Core. Pilates.

\section{Introdução}

Joseph Humbertus Pilates nasceu na Alemanha em 1880. Era uma criança doente que sofria de asma, raquitismo e febre reumática. Sua determinação em se tornar fisicamente mais forte o levou a estudar várias formas diferentes de movimentos durante toda sua vida (1).

Desde cedo ele decidiu contrariar a sua forma debilitada, buscando nas atividades físicas uma solução para vencer seus problemas. Ele utilizou yoga, mergulho, boxe, natação, esqui e conhecimentos de Fisiologia, Anatomia e Medicina Oriental. Até os movimentos animais não fugiram das suas observações (2). Em 1912, ele se mudou para a Inglaterra, dois anos antes da Primeira Guerra Mundial (3).

Joseph Pilates ganhava a vida na Inglaterra como lutador de boxe, e foi considerado um inimigo estrangeiro, sendo preso em um campo de concentração. Pilates tornou-se, então, enfermeiro e treinou os outros estrangeiros com os exercícios de cultura física que havia criado (4). Iniciou o uso das molas nas camas de hospital, desenvolvendo um sistema que inspirou a criação de seus equipamentos e de seu método (5).

Sua técnica foi reconhecida quando nenhum dos internos daquele campo sucumbiu a uma epidemia de gripe (influenza) que vitimou outras pessoas de outros campos (6).
Sem serem repetitivos ou extenuantes, os exercícios que ele propunha se adequavam às necessidades de cada um (7).

Após a guerra, Joseph retornou para a Alemanha, aperfeiçoando seu método com a Força Policial de Hamburgo. Mas, posteriormente, acabou se estabelecendo nos Estados Unidos, na cidade de Nova Iorque, abrindo um estúdio no mesmo edifício de New York City Ballet (8).

O seu método começou a ser praticado por importantes bailarinos que apresentavam lesões, sendo recuperados por Joseph Pilates. Logo, a técnica ganhou adeptos e se espalhou por todo o mundo.

Pilates denominava seu método de Contrologia ou Arte do Controle, que é a capacidade que o ser humano tem de se mover com conhecimento e domínio do próprio físico, apresentando uma completa coordenação do corpo, mente e espírito, utilizando princípios específicos para promover a integração entre eles, que são a concentração, centro de força (power house), fluidez, precisão, respiração e controle dos movimentos (9).

Independentemente da atividade, alguns elementos semelhantes sempre estão em jogo - estabilidade/ mobilidade, resistência/oposição, força de alavanca, articulação, balanceamento (10).

Os exercícios do método Pilates são, na sua maioria, executados na posição deitada, havendo diminui- 
ção dos impactos nas articulações de sustentação do corpo na posição ortostática e, principalmente, na coluna vertebral, permitindo recuperação das estruturas musculares, articulares e ligamentares particularmente da região sacrolombar $(11,12)$.

Contrologia introduziu a ideia de tratar a causa da doença e tentar evitar o problema por meio de atividades que geram bem-estar (11).

Dos 34 movimentos do método original resultaram cerca de 500 variações, realizadas com ou sem auxílio de aparelhos (9). Conforme Williams et al. (13) e Kuhnert (14), a frequência ideal para praticar Pilates deve ser três vezes por semana, em aulas de uma hora cada.

A técnica de Pilates consiste em dois tipos de aula: no solo (também denominado The Mat) e no aparelho (9). O trabalho tanto no solo quanto nos aparelhos (Cadeira, Reformer, Wall, Trapézio) consiste em exercícios para estabilizar pelve, controlar abdome, mobilizar articulações, fortalecer e alongar membros superiores e inferiores (15).

Baseando-se em princípios da cultura oriental, sobretudo relacionados às noções de concentração, equilíbrio, percepção, controle corporal e flexibilidade, e da cultura ocidental, destacando a ênfase relativa à força e ao tônus muscular, o Pilates provoca a tentativa do controle mais conscientemente possível dos músculos envolvidos nos movimentos (16).

Por meio das técnicas orientais que visam ao relaxamento, respiração, concentração, controle e flexibilidade, somadas à técnica ocidental, objetivando a ênfase no movimento com força, surgiu então a essência dos princípios do método Pilates (17).

0 interesse é que a atividade possa ser desenvolvida atendendo às necessidades específicas de cada praticante. Os exercícios são adaptados conforme as condições físicas do praticante, não havendo contraindicações. Há aqueles que podem ser evitados, e outros que se encaixam nas necessidades físicas do praticante (18).

A proposta de Joseph Pilates proporciona um amplo benefício para o corpo humano, estimulando a circulação, melhorando a flexibilidade, a amplitude de movimento, ou seja, a postura e o condicionamento do corpo (físico e mental) em geral (19).

Certamente, qualquer método mais abrangente e diversificado como o Pilates terá muitos princípios fundamentais. No caso do Método Pilates, são seis princípios-chave (20): centralização, concentração, controle, precisão, respiração e fluxo.
Os exercícios que compõem o método envolvem contrações isotônicas (concêntricas e excêntricas) e, principalmente, isométricas, com ênfase no que Joseph denominou power house (ou centro de força). Este centro de força é composto pelos músculos abdominais, transversoabdominal, multífido e músculos do assoalho pélvico, que são responsáveis pela estabilização estática e dinâmica do corpo. Então, durante os exercícios, a expiração é associada à contração dessas musculaturas e do diafragma (21).

No entanto, o método concentra-se no Centro de Força para fortalecer os músculos que foram utilizados incorretamente, ou que não foram usados (1).

A estabilidade do CORE é essencial para o equilíbrio de carga apropriado dentro da coluna vertebral, pélvis. 0 chamado núcleo assim como o grupo de músculos do tronco que envolvem a coluna vertebral e as vísceras abdominais. Abdominais, glúteos, cintura pélvica, paravertebrais e outros músculos trabalham em conjunto para proporcionar estabilidade espinhal (22).

0 termo "núcleo" é bastante abrangente e, por isso, comum entre praticantes e professores de atividades físicas. Outro nome para ele é "centro de força", usado por instrutores de Pilates para descrever os músculos coletivos do abdome, glúteos e músculos posteriores inferiores. Ao se exercitar, muitas pessoas não dão a devida atenção ao início de cada movimento. 0 resultado disso é que acabam se movimentando incorretamente e provocando danos (7).

Outro termo é a caixa definida por duas linhas horizontais: uma na altura dos ombros e outra em uma articulação do quadril até o outro. Com efeito, a caixa incorpora todo o tronco e pélvis. No conceito de centralização inclui-se o resto do tronco e, quando isso é feito, a caixa se torna um amplo centro do corpo humano (16).

Os programas de fitness incorporaram todas essas ideias; yoga, Pilates, Fit Ball, Roller e exercício funcional são exemplos práticos de atividades nas quais o CORE é acionado por meio de movimentos específicos para o complexo quadril-pélvico-lombar (23). 0 CORE é uma unidade integrada composta de 29 pares de músculos que suportam o complexo quadril-pélvico-lombar.

Dessa forma, percebe-se a importância dos resultados de estabilidade de ativação muscular altamente coordenada - envolvendo muitos músculos - e que os padrões de recrutamento devem mudar continuamente, dependendo da tarefa. Isso tem implicações 
sobre a prevenção da instabilidade e intervenções clínicas, fazendo com que os pacientes sejam aptos a sustentar situações de instabilidade (24).

Considerando o exposto, o presente estudo tem como objetivo geral analisar os aspectos relacionados à importância da estabilização central no método Pilates.

\section{Materiais e métodos}

Este estudo é uma revisão sistemática de literatura. É um tipo de investigação científica que tem por objetivo reunir, avaliar e conduzir uma síntese dos resultados de múltiplos estudos primários sobre o tema proposto aqui (25).

A busca pela literatura se deu por meio de uma pesquisa realizada na biblioteca central e virtual da Pontifícia Universidade Católica do Paraná e, também, no site de busca BIREME, acessando as bases de dados: LILACS - literatura latino-americana e do Caribe em ciências da saúde; MEDLINE - literatura internacional em ciências da saúde; PUB MED; CAPES. Os descritores utilizados para busca nas bases de dados supracitados foram pilates e core; tomando-se os devidos cuidados para confirmar a exatidão das informações apresentadas, a fim de descrever os conceitos e condutas mais adotadas pelos autores.

A busca nas bases de dados foi realizada no período entre a primeira e a segunda quinzena de agosto de 2010, e abrangeu livros, revistas e periódicos com base de dados científicos, e ano de publicação entre 2000 e 2010.

Após a leitura sistemática dos artigos, todo o material foi comparado a partir de uma leitura crítica de revisão bibliográfica.

A intenção desta revisão foi proporcionar ao leitor ampla variedade dos conceitos e condutas atuais da importância da estabilização central aos orientadores de Pilates.

\section{Resultados}

Neste trabalho foram incluídos 133 artigos, tendo uma avaliação eficiente que permitiu verificar a importância da estabilidade central no método Pilates. Os critérios de inclusão foram definidos com base no título que norteia a revisão e na observação de aspectos como amostra, instrumentos, aspectos éticos e período de publicação do artigo.

Entre os 192 artigos consultados, 133 abstracts se mostram pertinentes ao tema. Desses artigos, 60 versam sobre o método Pilates; 18 tratam de anatomia e fisiologia humana; e 55 discutem a estabilidade central e o CORE.

O método de Pilates é um programa completo de condicionamento físico e mental numa vasta órbita de exercícios potenciais. Os benefícios do método Pilates só dependem da execução dos exercícios com fidelidade aos seus princípios (26). Sua técnica foi reconhecida quando nenhum dos internos daquele campo sucumbiu a uma epidemia de gripe (influenza) que vitimou outras pessoas de outros campos (6).

0 programa de treinamento de estabilização central é criado para ajudar o indivíduo a obter ganhos de força, controle neuromuscular, potência e resistência muscular, com o objetivo de facilitar o funcionamento muscular equilibrado de toda a cadeia cinética (27).

A função do CORE é estabilizar a coluna e a pélvis durante os movimentos, manter um adequado alinhamento da coluna contra a ação da gravidade, localizar o nosso centro de gravidade, criar movimentos eficientes da cadeia cinética, propiciar uma base de suporte para os movimentos dos membros (origem do movimento), gerar força para os movimentos do tronco e prevenir lesões (28).

O CORE é uma unidade integrada, o sistema ativo é somente uma parte da unidade. Para que ele funcione de forma adequada, é preciso que haja uma coordenação eficiente entre os três sistemas (passivo, ativo e nervoso). Disfunções (alterações) em qualquer um dos três sistemas podem interferir negativamente na operação dos outros sistemas (29).

No treinamento do CORE, os músculos devem ser suficientemente fortes para estabilizar, mas também devem ser recrutados de forma apropriada. Tradicionalmente, o treinamento do CORE tem se caracterizado por treinar os grandes músculos globais. Negligenciar os músculos locais pode deixá-los "potencialmente" enfraquecidos e, assim, afetar a estabilidade da coluna (30).

Os benefícios do treinamento do CORE são: assegura um apropriado equilíbrio muscular, propicia a estabilidade, aumenta a força postural dinâmica e assegura o funcionamento apropriado da cadeia cinética por inteiro (31). Todos esses benefícios podem ser sumarizados em um único termo: eficiência neuromuscular (32). 
A capacidade do sistema neural e muscular permite que os agonistas, antagonistas, estabilizadores e sinergistas trabalhem de forma sinérgica no sentido de produzir e reduzir forças, assim como estabilizar a cadeia cinética em todos os três planos de movimento (33).

As cadeias musculares representam os circuitos contínuos por meio dos quais se propagam às forças organizadoras do corpo humano, buscando equilíbrio e economia energética para a realização das atividades do cotidiano, e tendo como prioridade a ausência da dor $(34,35)$. No entanto, ainda afirma-se que a existência dessa rede contínua de músculos favorece e também se mostra essencial nesse processo adaptativo das atividades cotidianas do homem (36).

0 treinamento de Estabilização Central, por sua vez, surgiu na década de 90 por meio da contribuição de inúmeros pesquisadores, como Gwen Jull, Paul Hodges, Carolyn Richardson, Julies Hides, Julie Sarhmann, entre muitos outros, no centro de pesquisa da Universidade de Queensland, em Brisbane Austrália, em associação a centros de pesquisa europeus. Esse treinamento visa ao controle postural e à manutenção do bom alinhamento do tronco, uma vez que se baseia no sinergismo com os músculos abdominais, para o equilíbrio da pelve - região composta pelos músculos profundos da pelve, coluna lombar e aqueles que cruzam a articulação coxofemoral (27).

No passado, o fortalecimento abdominal foi considerado a solução para problemas na região lombar ou enfraquecimento. Em 1950, Drs. Hans Krause e P. Williams desenvolveram um programa de condicionamento para os músculos abdominais (27).

Em 1980, o fisioterapeuta neozelandês Robin McKenzie desenvolveu uma sequência de exercícios para a região lombar e pélvica que enfatizavam os músculos posteriores do tronco.

0 método Pilates mostra-se eficiente quando é realizado um programa de exercícios com ênfase no fortalecimento da musculatura extensora do tronco, restaurando a função da coluna lombar e prevenindo o surgimento de lombalgia. Pode ser utilizado como estratégia para o fortalecimento dessa musculatura, atenuando o desequilíbrio entre a função dos músculos envolvidos na extensão e flexão do tronco (37).

Em um estudo, utilizou-se a ativação do controle do centro (cinturão abdomino-pélvico); ativação de grupos musculares específicos (abdome, adutores do quadril, períneo, multífidos e grande dorsal). Nele, afirma-se que o método Pilates é eficaz na redução do quadro álgico lombar apresentado durante a gestação. Houve o aumento gradativo das curvaturas fisiológicas, inerentes à gestação, melhora da consciência corporal e aumento da motivação em realizar atividades, relatadas pela paciente (38).

Pode-se observar o quanto o Pilates tem a contribuir para o equilíbrio corporal (físico e mental), auxiliando, assim, nas prevenções osteomioarticulares do corpo humano. Podemos subsidiar uma proposta significativa para a reeducação postural dos alunos de Pilates, e, acima de tudo, contribuir para uma melhor qualidade de vida (39).

0 método Pilates tem por característica, durante a execução dos seus movimentos específicos, a exigência de músculos abdominais, músculos estabilizadores da coluna vertebral, iliopsoas, quadríceps, e também da região lombossacral e do assoalho pélvico, o que talvez justifique a melhora considerável nestes testes que têm por características as valências envolvidas na sua execução, como força muscular nos membros inferiores, equilíbrio e surpreendentemente capacidade aeróbia. Isso tudo, possivelmente, em função de uma melhora no tônus muscular, o que diminui a fadiga muscular durante a realização dessas tarefas em menos tempo hábil (40).

\section{Considerações finais}

Embora seja necessária uma bibliografia mais abrangente, pode-se concluir que a estabilização central no Pilates é essencial para o equilíbrio apropriado de carga dentro da coluna vertebral, pélvis e cadeia cinética.

0 programa de treinamento de estabilização central foi criado para ajudar o indivíduo a obter ganhos de força, controle neuromuscular, potência e resistência muscular, visando facilitar o funcionamento muscular ao equilibrar toda a cadeia cinética. Um maior controle neuromuscular e força de estabilização oferecem um posicionamento biomecânico mais eficiente para toda a cadeia cinética, possibilitando, assim, a eficiência neuromuscular ideal ao longo desse sistema (26).

Se o objetivo é construir músculos, como no caso da musculação, ginástica localizada, com objetivos estéticos e de alta performance (body building), ou mesmo recuperação de uma lesão, situação em que um determinado grupo muscular necessita de atenção especial, os exercícios de isolamento muscular são os mais recomendados (41). 
De acordo com inúmeros autores, em virtude da dinâmica de execução, os exercícios de isolamento muscular priorizam o treinamento do "músculo", sendo frequentemente utilizados por inúmeras razões, entre elas: visa músculos específicos, de maneira isolada, performance de atletas, estética, corrigir desequilíbrios musculares, por exemplo, fortalecimento do manguito rotador e cintura escapular, estabilização pélvico lombar e do quadril, punho, cotovelo, joelho e tornozelo (42).

\section{Referências}

1. Gallagher SP, Kryzanowska R. O método pilates, de condicionamento físico. São Paulo: Competition; 2000.

2. Serpa D. Puxa e estica: Pilates, ginástica antiga que agora virou moda nos E.U.A., promete maravilhas. Revista Veja. 1998 [acesso 20 jul. 2011];89. Disponível em: http://veja.abril.com.br/270598/p_089.html.

3. Ribeiro M. Pilates, equilíbrio de corpo e mente. Jornal da Orla; 2004:3.

4. Camarão T. Pilates com bola no Brasil. Rio de Janeiro: Alegro; 2005.

5. Sacco ICN, Andrade MS, Souza PS, Nisiyama M, Cantuária AL, Maeda FYI, et al. Pilates method in review: biomechanical aspects of specific movements for postural reorganization - Cases report. R Bras Ci Mov. 2005; 13(4):65-78.

6. Herdman A, Selby A. Pilates: como criar o corpo que você deseja. São Paulo: Manole; 2000.

7. Ungaro A. A promessa de pilates. São Paulo: Caras; 2005.

8. Hayashida W. O que é Pilates? São Paulo, 2005. [acesso 10 ago. 2010]. Disponível em: www.wmulher.com.br.

9. Borges J. Princípios básicos do método Pilates. Módulo, 2004.

10. Siler B. Desafios do corpo pilates. Tradução de Angel Santos. São Paulo: Summus; 2009.

11. Pilates JH. The complete writings of Joseph H. In: Sean P, Gallagher PT, Romana K, editors. Pilates: Return to life through contrology and your health. Philadelphia: Bain Bridge Books; 2000.
12. Gallagher SP, Kryzanowska R. The Pilates ${ }^{\circledR}$ method of body conditioning. Philadelphia: Bain Bridge Books; 1999.

13. Williams S, Jasen D. Para começar a praticar Pilates. São Paulo: Publifolha; 2005.

14. Kuhnert C. Um corpo perfeito com Pilates. São Paulo: Vitória Régia, 2002.

15. Nogueira J. Método Pilates ganha espaço. J Commercio. 2002:20. Coluna Família.

16. Muscolino JE, Ciprini S. Pilates and "Powerhouse". J Body Mov Ther. 2004;8:15-24.

17. Craig C. Pilates com a bola. 2a ed. São Paulo: Phorte; 2005.

18. Abrami MCR, Browne RG. Material didático do curso de formação CGPA Pilates. São Paulo: Manole; 2003.

19. Menegatti F. Pilates: força e flexibilidade. Rev Muscle in Form. 2004;6:42.

20. Liekens, B. The Pilates Studio Teacher Training Manual. Part IFBasic/Intermediate. New York: The Pilates Studio; 1997.

21. Silva ACLG, Mannrich G. Pilates na reabilitação: uma revisão sistemática. Fisioter Mov. 2009;22(3):449-55.

22. Akuthota V, Ferreiro A, Moore T, Fredericson M. Core stability exercise principles. Curr Sports Med Rep. 2008;7(1):39-44.

23. Guiselini M. Multifuncional Exercise Training. 2009. [cited 2011 jun. 15]. Available at: http://www.institutomauroguiselini.com.br/?s\&tubepress_page $=10$.

24. McGill SM , Grenier S, Kavcic N, Cholewicki J. Coordination of muscle activity to assure stability of the lumbar Spine. J Electromyogr Kinesiol. 2003;13(4):353-9.

25. Cook DJ, Mulrow CD, Haynes RB. Systematic reviews: synthesis of best evidence for clinical, decisions. Ann Intern Med. 1997;126(5):376-8.

26. Miranda LB, Morais PDC. Efeitos do método Pilates sobre a composição corporal e flexibilidade. Rev Bras Prescr Fisiol Exerc. 2009;3(13):16-21.

27. Clark MA. Treinamento de estabilização central em reabilitação. In: Prentice WE, Voight ML. Técnicas em reabilitação musculoesqueléticas. Porto Alegre: Artmed; 2003. p. 245-63. 
28. O’Sullivan P. Lumbar segmental 'instability': clinical presentation and specific stabilising exercise management. Man Ther. 2000;5(1):2-12.

29. Panjabi M. The stabilising system of the spine. Part 1. Function, disfunction, adaptation and enhancement. J Spinal Disord. 1992;5(4):383-9.

30. Houglum P. Therapeutic exercise for musculoskeletal injuries. 2nd ed. Champaign: Human Kinetics Publishers; 2005.

31. Beach P. Personal communication. Lecturer: British College of Osteopathic Medicine; British School of Osteopathy; 2003.

32. Comerford MS. Functional stability re-training: principles and strategies for managing mechanical dysfunction. Man Ther. 2001;6(1):3-14.

33. Lee D. An integrated model of joint function and its clinical application. Proceedings of $4^{\text {th }}$ Interdisciplinary World Congress on Low Back \& Pelvic Pain. Montreal, Canada; 2001. p. 137-52.

34. Busquet L. As cadeias musculares: tronco, coluna cervical e membros superiores. Belo Horizonte: Busquet; 2001.

35. Marques AP. Cadeias musculares. 2a ed. São Paulo: Manole; 2005.

36. Busquet L. As cadeias musculares: lordoses, cifoses, escolioses e deformações torácicas. Belo Horizonte: Busquet; 2001.

37. Kolyniak IEG, Cavalcanti SMB, Saldanha M. Avaliação isocinética da musculatura envolvida na flexão e extensão do tronco: efeito do método Pilates. Rev Bras Med Esporte. 2004;10(6):487-90. doi.org/10.1590/ S1517-86922004000600005
38. Machado CG, Araújo TG, Sandoval RA, Machado CANR, Freitas MS. O método pilates na diminuição da dor lombar em gestantes. Rev Elet Faculd Montes Belos 2008;3(1).

39. Mattos R, Samano T. Intervenção do pilates na reabilitação na reeducação postural: um estudo de caso. [acesso 15 jun. 2011]. Disponível em: http://bioequilibriopilates.com/blog/?cat=9

40. Curi VS. A influência do método Pilates nas atividades de vida diária de idosas. (dissertação). Porto Alegre: Pontifícia Universidade Católica do Rio Grande do Sul; 2009.

41. Check P. Functional Training: what, when, why, how and where to do it. 2008. (cited 2011 jun. 15]. Available at: http://www.ppssuccess.com/FoodforThought/ ArticlesbyPaul/ArticlesbyPaulChekDetailPage/tabid/ 496/smid/2144/ArticleID/42/reftab/104/Default. aspx.

42. Prentice WE. Rehabilitation techniques for sports medicine \& athletic training. 4th ed. New. York: McGrawHill; 2004.
Recebido: 20/10/2011

Received: 10/20/2011

Aprovado: 21/03/2012

Approved: 03/21/2012 